\title{
XXXIII. On the constitution of the surface layers of liquids-Part I
}

\section{S.A. Shorter D.Sc.}

To cite this article: S.A. Shorter D.Sc. (1916) XXXIII. On the constitution of the surface layers of liquids-Part I, Philosophical Magazine Series 6, 31:184, 260-275, DOI: $10.1080 / 14786440408635500$

To link to this article: http://dx.doi.org/10.1080/14786440408635500

曲 Published online: 08 Apr 2009.

Submit your article to this journal $[\pi$

Џll Article views: 2

Q View related articles $₫$ 
Thus the transmission through the crystal had not produced a deflexion of $\cdot 012$ millimetre at a distance of 140 (about) centimetres from the refracting prism ; that is, the deviation was less than 2 seconds of arc. We conclude from this that the refractive index of potassium bromide for radiation of wave-length in the neighbourhood of $5 \times 10^{-8} \mathrm{~cm}$. is between 0.999995 and 1.000005 *.

It is of course possible that if only radiation of frequency within certain narrow limits were refracted appreciably, the intensity of this would be toc small to produce a distinguishable feature on the photographic plate.

Experiments with more nearly homogeneous beams of radiation might indicate such refraction for a particular wave-length. Although this is a possibility, there is little reason for expecting that such experiments would lead to results differing from those already obtained. For the radiations used in these experiments covered a great portion of what may be regarded as the $K$ absorption band associated with the bromine " $\mathrm{K}$ " radiation.

I wish to express my thanks to Mr. J. H. Hagger,now Inspector of Munitions-for his assistance in these experiments.

XXXIII. On the Constitution of the Surface Layers of Liquids. By S. A. ShonTer, D.Sc., Assistant Lecturer in Plysics in the University of Leeds.-Part I. †

Contents.

§1. Introduction.

2. The General Case of a Binary System.

$\$ 3$. 'The Principle of the Relativity of the Surface Magnitudes. $\$ 4$. Special Cases of a Binary System.

\section{$\$ 1$. Introduction.}

THE principles of thermodynamics were first applied to 1 the question of the constitution of the heterogeneous layer separating two phases, by Gibbs in his well-known work "On the Equilibrium of Heterogeneous Substances" $\neq$. As in the case of many other extremely interesting problems, Gibbs's mode of treatment of the subject presents mathematical difficulties, which are insuperable to practically all of those to whom the subject is of interest. The object of

* Bromine $\mathrm{K}$ radiation has a wave-length about $10^{-8} \mathrm{~cm}$.

+ Communicated by the Author.

† Scientific Papers, vol. i. p. 235. 
the present work is to develop the thermodynamical theory of the subject in an elementary manner, preserving at the same time perfect mathematical rigour and the utmost generality of treatment.

The first attempt at an elementary exposition of the subject was made by Milner*, who obtained a formula for the "surface excess" of a solute, applicable to the case of a dilute solution in contact with a third substance.

The theory developed in the present work is applicable to solutions of any degree of concentration-and therefore to the case of a mixture of two perfectly miscible liquids. Since in this latter case the distinction between solvent and solute is a purely arbitrary one, the theory must take account of the "surface excess" of both solvent and solute. This consideration of the "surface excess" of both components of a solution leads to a curious and interesting principle, which I bave termed the "Principle of the Relativity of the Surface Magnitudes." This principle underlies all Gibbs's theory, though it is never explicitly referred to in it.

\section{\$2. The General Case of a Binary System.}

We will first consider the case of the interface separating two phases of a system containing two components. This general case includes a number of special cases, which we will consider in $\S 4$ of the present paper.

The most elementary method of treating the subject is to consider the working of an ideal "engine" in which the contractile tendency of the surface and the expansive tendency of the volume are both used as a means of producing an external mechanical effect. Such an engine is represented in fig. 1. $Q$ is a vessel of rectangular horizontal cross-section, containing masses $M_{0}$ and $M_{1}$ of two components $\mathrm{C}_{0}$ and $\mathrm{C}_{1}$ respectively, in two phases $\phi$ and $\phi^{\prime}$ separated by a plane surface of area $\sigma$ and tension $\tau$. The area of the surface may be varied by means of a movable plate $P$, which fits accurately to the sides of the vessel parallel to the plane of the paper. The horizontal portion of this plate passes through a slot in one of the vertical sides perpendicular to the plane of the paper. This side is sufficiently thick in the neighbourhood of the slot for the plate to be incapable of any motion but a horizontal motion of translation. The volume $v$ of the system may be varied by means of the piston $\mathrm{R}$. 
Suppose now that the engine goes through a reversible isothermal cycle. According to the flrst law of thermodynamics, the total work done by the system is equal to the

Fig. 1.

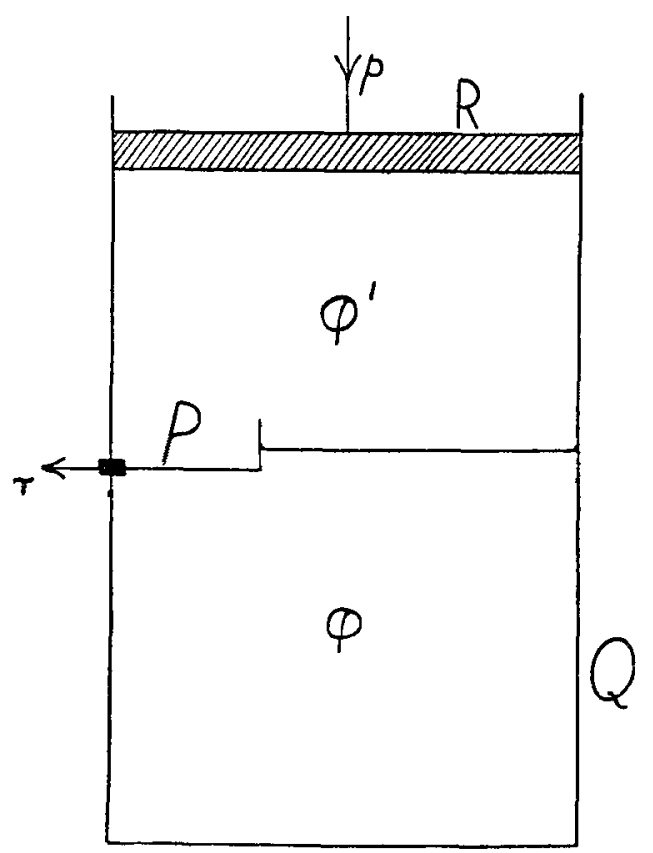

total heat absorbed by it. According to the second law the total heat absorbed is zero. Hence the total work done is zero.

Let the cycle consist of the following four stages :-

(1) The surface-tension being maintained at the constant value $\tau$, let the volume $v$ of the system be changed till the surface increases from $\sigma$ to $\sigma+\delta \sigma$. During this change the pressure $p$ will remain constant.

(2) The area of the surface being maintained constant, let the pressure $p$ be changed till the surface. tension changes from $\tau$ to $\tau+\delta \tau$.

(3) The surface tension being maintained constant, let the volume be changed till the area of the surface changes from $\sigma+\delta \sigma$ to $\sigma$.

(4) The area of the surface being maintained constant, let the pressure be changed till the surfacetension changes from $\tau+\delta \tau$ to $\tau$. 
If we take values of $\sigma$ as abscissæ and values of $\tau$ as ordinates, the cycle is represented by the elementary rectangle PQRS of area $\delta \sigma \delta \tau$, described in an anti-clockwise

Fig. 2.

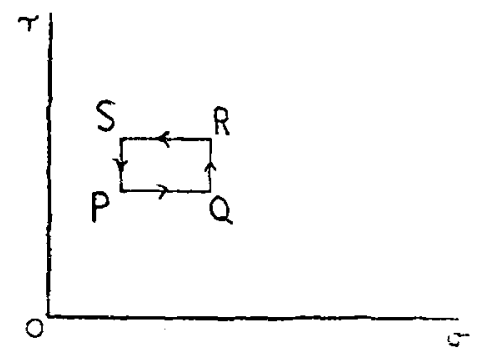

direction (see fig. 2). Hence the system does an amount lof work $\delta \sigma \delta$ r against the force controlling the surface *. The force controlling the volume must therefore do an equal amount of work on the system. Suppose now that we take

Fig. 3.

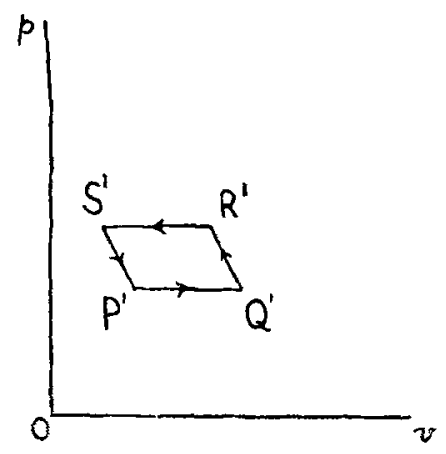

values of $v$ as abscisse and values of $p$ as ordinates. The cycle will be represented by a parallelogram $\mathrm{P}^{\prime} \mathrm{Q}^{\prime} \mathrm{R}^{\prime} \mathrm{S}^{\prime}$ (se日 fig. 3) with two sides $\left(\mathrm{P}^{\prime} \mathrm{Q}^{\prime}\right.$ and $\left.\mathrm{R}^{\prime} \mathrm{S}^{\prime}\right)$ parallel to the $v$ axis,

* A certain force will be neeessary to counteract the effect of the difference in the hydrostatic pressure on the two sides of the vertical portion of the plate $\mathbf{P}$. Sines the work done by this force in any displacement is equal to the product of the total weight of the components and the increase in the height of their centre of gravity, the work done in a cycle will be zero, so that this force may be left out of account in considering a cycle of operations. 
of length $(d v / d \sigma)_{\theta \tau} \delta \sigma$, a distance $(d p / d \tau)_{\theta} \delta \tau$ apart. Now the perimeter must be described in an anti-clockwise direction. Hence the above two differential coefficients must have the same sign; so that equating the areas of the parallelograms $P Q R S$ and $P^{\prime} Q^{\prime} R^{\prime} S^{\prime}$, we obtain the relation

$$
\left(\begin{array}{l}
d v \\
d \sigma
\end{array}\right)_{\theta \tau}=\left(\frac{d \tau}{d p}\right)_{\theta} \cdot \text {. . . . . . }
$$

Since in a binary system the constancy of $\tau$ and $\theta$ is equivalent to the constancy of $p$ and $\theta$, this equation may be written *

$$
\left(\frac{d v}{d \sigma}\right)_{p \theta}=\left(\frac{d \tau}{d p}\right)_{0} \ldots . . . .
$$

We see from this equation that if the surface-tension depends upon the pressure, it is necessary to change the volume, when the area of the surface is changed, in order to keep unchanged the nature of the different parts of the system. Such a change would obviously be unnecessary if the two phases were homogeneous right up to the surface of separation. Let $\rho_{0}$ and $\rho_{1}$ denote the densities in the $\phi$ phase, and $\rho_{0}^{\prime}$ and $\rho_{1}{ }^{\prime}$ those in the $\phi^{\prime}$ phase, of $\mathrm{C}_{0}$ and $\mathrm{C}_{1}$ respectively. Let $V^{\prime}$ and $V^{\prime}$ denote the respective volumes of the phases, and $M_{0}$ and $M_{1}$ the respective total masses of the components. We see that in general the equations

$$
\begin{aligned}
& M_{0}=\rho_{0} V+\rho_{0}^{\prime} V^{\prime}, \\
& M_{1}=\rho_{1} V+\rho_{1}^{\prime} V^{\prime},
\end{aligned}
$$

will not be verified. Let us write

$$
\begin{aligned}
& \sigma \Gamma_{0}=\mathbf{M}_{0}-\left(\rho_{0} \mathbf{V}+\rho_{0}{ }^{\prime} \mathbf{V}^{\prime}\right), . . \cdot \\
& \sigma \Gamma_{1}=\mathbf{M}_{1}-\left(\rho_{1} \mathbf{V}+\rho_{1}^{\prime} \mathbf{V}^{\prime}\right) . . .
\end{aligned}
$$

We will call (following Milner $\dagger$ ) each of the quantities $\Gamma_{0}$ and $\Gamma_{1}$ (defined by the above equations) the "surface excess" of the corresponding component. It is evident that $\Gamma_{0}$ and $\Gamma_{1}$ will depend only on the nature of the system, and not on the dimensions of its parts.

The differential coefficient $(d v / d \sigma)_{\theta p}$ is easily evaluated in

* This relation may be deduced more concisely, though iu a less elementary manner, from the equation $d \mathrm{U}=\theta d \mathrm{~S}-p d v+\tau d \sigma$ (U=energy, $\mathrm{S}=$ entrop $v)$, br writing it in the form $d(\mathrm{U}-\theta \mathrm{S}+p v)=-\mathrm{S} d \theta+r d p+\tau d \sigma$.

$\dagger$ Loc. cit. Gibls uses the term "surface density." 
terms of $\Gamma_{0}, \Gamma_{1}$ and the densities. Since the constancy of $p$ and $\theta$ involves the constancy of $\rho_{0}, \rho_{0}{ }^{\prime}, \rho_{1}, \rho_{1}{ }^{\prime}, \Gamma_{0}$ and $\Gamma_{1}$, we have from equations (3) and (4)

Since

$$
\Gamma_{0} d \sigma+\rho_{0} d V+\rho_{0}^{\prime} d V^{\prime}=0
$$

and

$$
\Gamma_{1} d \sigma+\rho_{1} d \mathbf{V}+\rho_{1}^{\prime} d \mathbf{V}^{\prime}=0 . \quad . \quad .
$$

we have

$$
v=\mathrm{V}+\mathrm{V}^{\prime} \cdot \text {. . . . . . }
$$

$$
d v=d \mathrm{~V}+d \mathrm{~V}^{\prime} \ldots . . . \quad .
$$

From equations (5), (6), and (8) we readily obtain the desired relation

$$
\left(\frac{d v}{d \sigma}\right)_{\theta p}=\frac{\Gamma_{1}\left(\rho_{0}-\rho_{0}^{\prime}\right)-\Gamma_{0}\left(\rho_{1}-\rho_{1}^{\prime}\right)}{\rho_{0}^{\prime} \rho_{1}-\rho_{0} \rho_{1}^{\prime}} . .
$$

Substituting this value in equation (2) we obtain the equation

$$
\frac{\Gamma_{1}}{\rho_{1}-\rho_{1}^{\prime}}-\frac{\Gamma_{0}}{\rho_{0}-\rho_{0}^{\prime}}=\frac{\rho_{0}{ }^{\prime} \rho_{1}-\rho_{0} \rho_{1}^{\prime}}{\left(\rho_{0}-\rho_{0}^{\prime}\right)}\left(\frac{d \tau}{\left(\rho_{1}-\rho_{1}^{\prime}\right)}\left(\frac{d \tau}{d p}\right)_{\theta} .\right.
$$

This is the most general relation possible (in the case of a binary system) between the surface magnitudes and quantities capable of experimental determination.

\section{\$. The Principle of the Relativity of the Surface Magnitudes.}

It will be seen that in the case of a binary system our theory gives only one equation connecting the two quantities $\Gamma_{0}$ and $\Gamma_{1}$ with quantities capable of experimental determination. We cannot obtain separate equations for $\Gamma_{0}$ and $\Gamma_{1}$. At first sight, this seems to imply some incompletentess or imperfection in the thermodynamical theory. A closer examination of what is meant by the term "surface excess" shows, however, that this idea is wrong, and that the theory yields all that could be expected of it.

Suppose that we take as abscissæ the distances of points from some reference plane parallel to the surface, and as ordinates the values of the density of a component. The graph will consist of two straight lines parallel to the axis of abscissæ (corresponding to the interiors of the two phases) joined by a curved line (corresponding to the region of 
heterogeneity). A simple type of such a curve is shown in fig. 4. It is evident that the position of the surface of

Fig. 4.

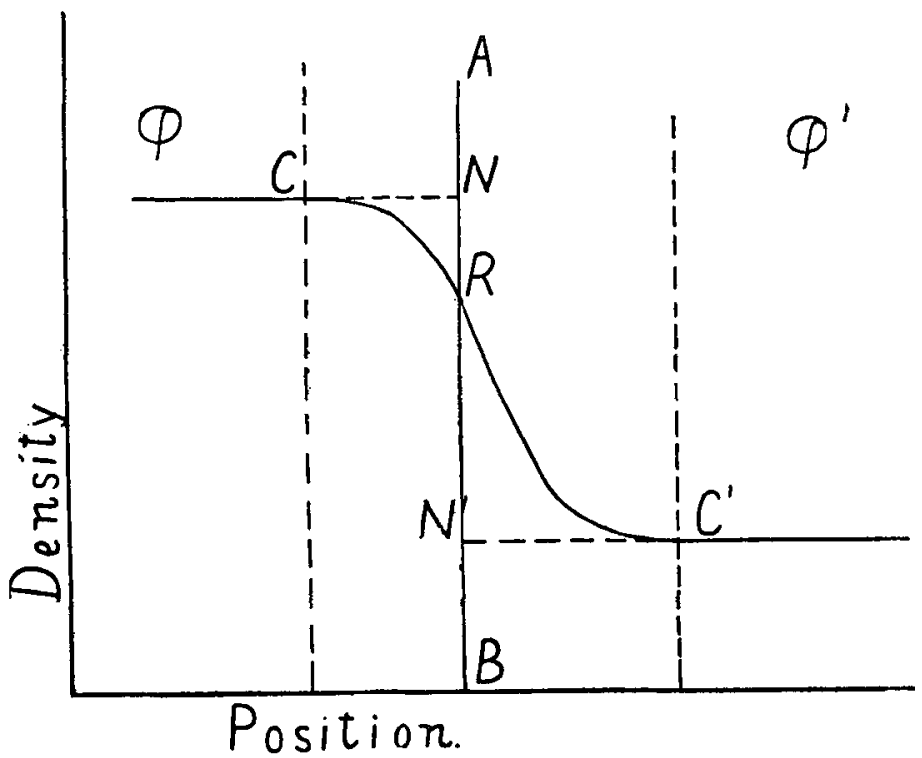

separation may be assigned quite arbitrarily within the limits of the region of heterogeneity. If we assign a position $A B$, the surface excess is given by

$$
\Gamma=\text { area } C^{\prime} N^{\prime} R \text {-area CNR. }
$$

The value of $\Gamma$ may vary within wide limits with the arbitrarily assigned position of the surface of separation.

If we choose the position of $\mathrm{AB}$ so that

$$
\text { area } C N R=\text { area } C^{\prime} N^{\prime} R \text {, }
$$

the surface excess is zero. We will call the plane, relative to which the surface excess of a component is zero, the "zero-plane" of that component. If the density assumes in the surface-layer values greater than in either phase, the position of the "zero-plane" is fixed (see fig. 5) by the relation

$$
\text { area } C E D+\text { area } C^{\prime} N^{\prime} R=\text { area } R D N \text {. }
$$

If the increase in density in the surface-layer is very great the "zero-plane" may lie in the interior of one of the phases (as in fig. b). 
of the Surface Layers of Liquids.

Fig. 5.

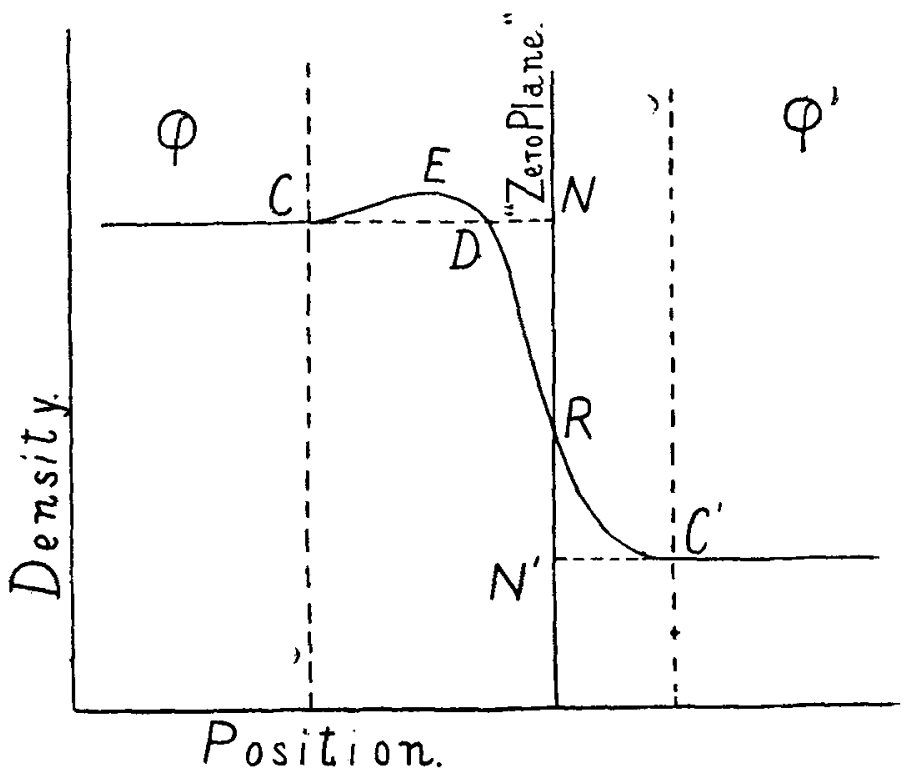

Fig. 6.

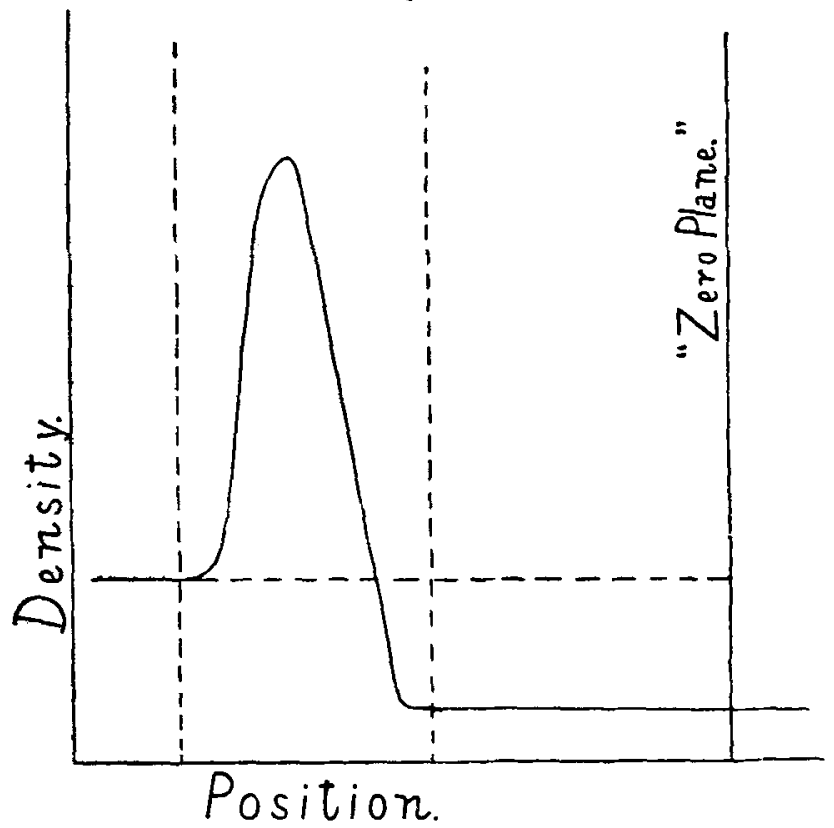


The value of the surface excess relative to a plane a distance $\approx$ from the "zero-plane" in the direction of the $\phi$ phase is evidently given by

$$
\Gamma=\left(\rho-\rho^{\prime}\right) z
$$

Consider now a system of two components $C_{0}$ and $C_{2}$. The "zero-plane" of each component will have a definite position, and the distance between the two will have a definite value, characteristic of the system. Suppose now that $\Gamma_{0}$ and $\Gamma_{1}$ are the respective values of the surface excesses, relative to a plane whose distances from the "zero-planes" of $\mathrm{C}_{0}$ and $\mathrm{C}_{1}$, measured in the direction of the $\phi$ phase, are $z_{0}$ and $z_{1}$ respectively. We then have

$$
\text { and } \quad \begin{array}{ll}
\Gamma_{0} & =\left(\rho_{0}-\rho_{0}{ }^{\prime}\right) z_{0} \\
& \\
& \Gamma_{1}=\left(\rho_{1}-\rho_{1}\right) z_{1} .
\end{array}
$$

Hence, if $h$ denote the distance of the "zero-plane" of $\mathrm{C}_{1}$ from that of $\mathrm{C}_{0}$ measured in the direction of the $\phi^{\prime}$ phase, we have

$$
h=\hat{z}_{1}-z_{0}=\frac{\Gamma_{1}}{\rho_{1}-\rho_{1}^{\prime}}-\frac{\Gamma_{0}}{\rho_{0}-\rho_{0}^{\prime}} .
$$

Referring back to equation (10)-the general equation for a binary system-we see at once the reason for the apparent incompleteness of our theory and the particular form of the equation. The quantities $\Gamma_{0}$ and $\Gamma_{1}$ are purely arbitrary magnitudes which could not possibly appear singly in any thermodynamical relation. They may, however, be combined in such a way as to yield a quantity which is not arbitrary, and this quantity appears on the left-hand side of equation (10), which may be written in the form

$$
h=\frac{\rho_{0}^{\prime} \rho_{1}-\rho_{0} \rho_{1}^{\prime}}{\left(\rho_{0}-\rho_{0}{ }^{\prime}\right)\left(\rho_{0}-\rho_{1}^{\prime}\right)}\left(\frac{d \tau}{d p}\right)_{\theta} \cdot \cdot \cdot .
$$

We may, however, interpret equation (10) in another way. The value of the surface excess of $\mathrm{C}_{1}$ relative to the "zero* plane" of $\mathrm{C}_{0}$ is evidently $\left(\rho_{1}-\rho_{1}^{\prime}\right) h$ or

$$
\Gamma_{1}-\frac{\rho_{1}-\rho_{1}^{\prime}}{\rho_{0}-\rho_{0}^{\prime}} \Gamma_{0}
$$

Let us adopt the notation of Gibbs and use the symbol 
$\Gamma_{r(s)}$ to denote the surface excess of any component $\mathrm{C}_{r}$ in a system relative to the "zero-plane" of another component $\mathrm{O}_{s}$. Equation (10) may then be written

$$
\Gamma_{1(0)}=\frac{\rho_{0}^{\prime} \rho_{1}-\rho_{0} \rho_{1}^{\prime}}{\rho_{0}=\rho_{0}^{\prime}}\left(\frac{d \tau}{d p}\right)_{\theta} \cdot \ldots . .
$$

The value of the surface excess of $\mathrm{C}_{0}$ relative to the "zeroplane" of $\mathrm{C}_{1}$ is evidently equal to $-\left(\rho_{0}-\rho_{0}{ }^{\prime}\right) h$ or

$$
\Gamma_{0}-\frac{\rho_{0}-\rho_{0}{ }^{\prime}}{\rho_{1}-\rho_{1}} \Gamma_{1}
$$

so that equation (10) may be written

$$
\Gamma_{0(1)}=-\frac{\rho_{0}^{\prime} \rho_{1}-\rho_{0} \rho_{1}^{\prime}}{\rho_{1}-\rho_{1}^{\prime}}\left(\frac{d \tau}{d p}\right)_{\theta} \cdot \quad . \quad .
$$

The above general considerations apply, of course, to a system containing any number of components. Thus in the case of a system of $n$ components, there are $n$ "zeroplanes," and the theory should yield $n-1$ equations for the $n-1$ lengths which fix the relative positions of these planes.

In recent years the surface excess of a solute in a binary liquid mixture has been determined experimentally by observing the change of concentration caused by a large extension of surface. We have seen that the surface excess of a component is a purely arbitrary magnitude, so that the interesting question arises as to what is given by the above experiment. The only case which has been investigated practically is that of a solution in contact with a third substance. This case will be considered in Part II. of the present work. We will consider here an imaginary experiment with a two-phase binary system, which could not be realized in practice because of the smallness of the changes involved, but which will serve to illustrate the point in question. Suppose that a closed vessel contains a binary liquid mixture in contact with the vapour phase. Let the vessel be tilted so that the area of the surface separating the phases is increased from $\sigma$ to $\sigma-\Delta \sigma$. This will in general cause the vapour pressure to alter. Suppose that by the addition of an amount $\Delta \mathrm{M}_{1}$ of the solute $\mathrm{C}_{1}$ the initial pressure (and therefore the initial concentration of the solution) is restored. One would at once say that the surface excess of the solute is $\Delta \mathrm{M}_{1} / \Delta \sigma$. Let us examine 
the question in the light of the above theory. Initially we have

and, finally,

$$
\begin{aligned}
& M_{0}=\rho_{0} V+\rho_{0}^{\prime} V^{\prime}+\Gamma_{0} \sigma, \\
& M_{1}=\rho_{1} V+\rho_{1}^{\prime} V^{\prime}+\Gamma_{1} \sigma,
\end{aligned}
$$

$$
\begin{aligned}
M_{0} & =\rho_{0}(V+\Delta V)+\rho_{0}^{\prime}\left(V^{\prime}+\Delta V^{\prime}\right)+\Gamma_{0}(\sigma+\Delta \sigma), \\
M_{1}+\Delta M_{1} & =\rho_{1}(V+\Delta V)+\rho_{1}^{\prime}\left(V^{\prime}+\Delta V^{\prime}\right)+\Gamma_{1}(\sigma+\Delta \sigma) ;
\end{aligned}
$$

and since the total volume is constant

$$
\Delta V+\Delta V^{\prime}=0 \text {. }
$$

From these equations we readily find that

$$
\frac{\Delta \mathrm{M}_{1}}{\Delta \sigma}=\Gamma_{1}-\frac{\rho_{1}-\rho_{1}^{\prime}}{\rho_{0}-\rho_{0}^{\prime}} \Gamma_{0}=\mathrm{I}_{1(0)}^{\prime}, \quad . \quad .
$$

i.e. that the value of the surface excess of $\mathrm{C}_{1}$ deduced from the experimental data is the value relative to the "zeroplane" of $\mathrm{C}_{0}$.

\section{\$4. Special Cases of a Binary System.}

Case I. Two Immiscible Liquids.

The simplest case of a binary system is that in which each phase consists of a single substance, $i$. e. that formed by two immiscible liquids. Let us supposs that the $\phi$ phase contains only the component $\mathrm{C}_{0}$, and the $\phi^{\prime}$ phase only the component $\mathrm{C}_{1}$. Equation (10) then takes the form

$$
\frac{\Gamma_{1}}{\rho_{1}^{\prime}}+\frac{\Gamma_{0}}{\rho_{0}}=-\left(\frac{d \tau}{d p}\right)_{\theta} \cdot . . . .
$$

This equation may also be written in the forms

$$
\Gamma_{1(0)}=-\rho_{1}\left(\frac{d \tau}{d p}\right)_{\theta} \quad . \quad . \quad . \quad . \quad .
$$

and

$$
\Gamma_{0(1)}=-\rho_{0}\left(\frac{d \tau}{d p}\right)_{\theta} \cdot \cdots \cdot . \quad . \quad .
$$

This result is of importance in relation to the question of the composition of the surface-layers of pure liquids. It is 
often asserted that the surface-layers of a liquid are in a state of compression *. According to Lewis $t$, who has developed a quantitative theory of this supposed surface compression, the variation of the densities of two immiscible liquids in the neighbourhood of the surface

Fig. 7.

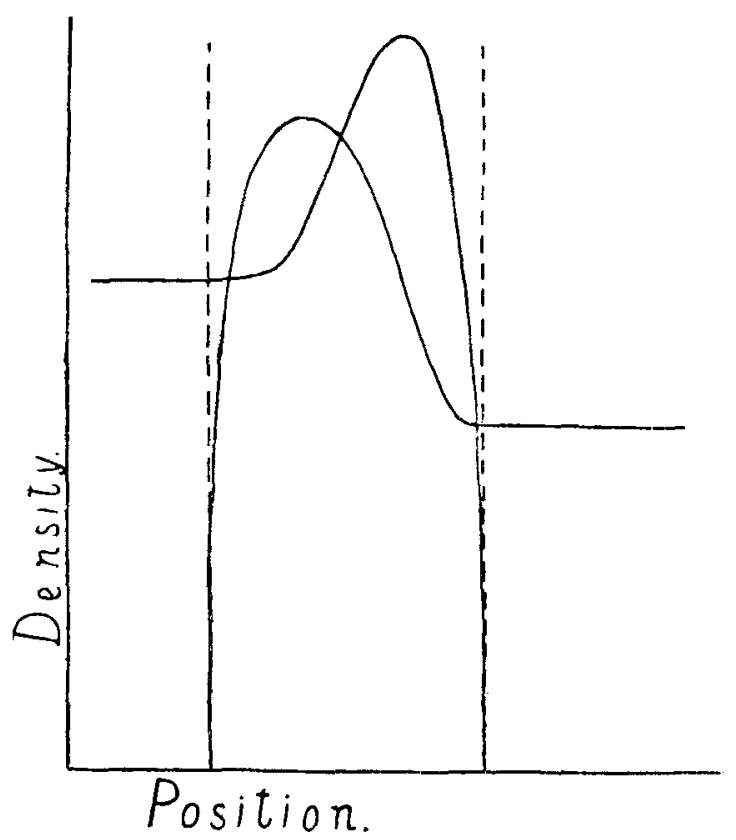

separating them is as shown in fig. 7. In the case of water it is calculated that the mean density in the surfacelayer is about twice that in the body of the liquid. An effect of the same order of magnitude is calculated for a number of other liquids. In the exceptional case of mercury the mean density in the surface-layer is only 84 of the bulk density.

Let us examine this theory of surface compression in the

* See, for example, 'The Chemistry of Colloids,' by W. W. Taylor (Arnold, 1915), pp. 229 \& 250.

+ Phil. Mag. Sept. 1910, p. 502 ; Kolloid-Zeitschrift, vol. vii. p. 197 (1910). 
light of the thermodynamical theory given above. Equation (15) may be written in the form

$$
h=\left(\frac{d \tau}{d p}\right)_{\theta} \cdot \text {. . . . . . }
$$

where $h$, it will be remembered, is the distance of the "zero-plane" of $\mathrm{C}_{1}$ from that of $\mathrm{C}_{0}$ measured in the direction of the $\phi^{\prime}$ phase. Now in the case of a system having a density diagram of the kind shown in fig. $7, h$ is evidently negative-as is also the case if only one of the liquids exhibits surface compression. Hence the theory of surface compression leads to the conclusion that the tension of the interface separating two immiscible liquids is diminished by increase of pressure. This result may, of course, be deduced from quite elementary considerations. The change of pressure produced by an extension of the interface at constant volnme must be such as to increase the tension. If the liquids are compressed in the surface-layers this extension produces a diminution of pressure so that $d \tau / d p$ is negative.

The effect of pressure on the interfacial tension has been investigated by Lynde* in the case of a number of pairs of immiscible or partially miscible liquids. In the case of water and mercury it is found that the interfacial tension is increased $\cdot 74$ per cent. by an increase of pressure of $5000 \mathrm{lb}$. per sq. inch. This gives (assuming $\tau=370 \mathrm{dyne} / \mathrm{cm}$.) for $d \tau / d p$ the value $8.2 \times 10^{-9} \mathrm{~cm}$. or $\cdot 082 \mu \mu$.

This result is, of course, inconsistent with the view that either the water or the mercury are compressed in the surface-layer. It is interesting to calculate the order of magnitude of $d \tau / d p$ required by Lewis's theory. Since the mercury does not suffer any superficial increase of density its "zero-plane" will be situated somewhere in the surfacelayer. Since the mean density of the water in the surfacelayer is double the bulk density, the "zero-plane" of the water will lie in the interior of the mercury, a distance from the nearer boundary of the surface-layer equal to the thickness of the surface-layer. We therefore have

$$
-\frac{d \tau}{d p}>\text { thickness of surface-layer. }
$$

If we assume the value $10^{-6} \mathrm{~cm}$. for the thickness of the

* Phys. Rev. vol. xxii. p. 181 (1906). 
surface-layer, we obtain a value of $d \tau / d p$ opposite in sign to, and arithmetically more than a hundred times as large as, the value obtained experimentally.

A similar discrepancy between theory and experiment exists in the case of the ether-mercury interface, Lynde's experiments giving for $d \tau / d p$ a small positive value, and Lewis's theory a relatively large negative value. Our theory shows, therefore, that the theory of surface compression is inconsistent with experimental data relating to the effect of pressure on the interfacial tension.

Case II. Two Partially Miscible Liquids.

Suppose that the two phases are the liquid layers formed by two partially miscible liquids, the $\phi$ phase being the $\mathrm{C}_{0}$ layer. If we write $c_{0}=\rho_{0}{ }^{\prime} / \rho_{0}$ and $c_{1}=\rho_{1} / \rho_{1}^{\prime}$, we have

or

$$
\begin{aligned}
& \Gamma_{1(0)}=-\frac{\rho_{1}{ }^{\prime}\left(1-c_{0} c_{1}\right)}{1-c_{0}}\left(\frac{d \tau}{d p}\right)_{\theta} \cdot . . \\
& h=\frac{1-c_{0} c_{1}}{\left(1-c_{0}\right)\left(1-c_{1}\right)}\left(\frac{d \tau}{d p}\right)_{\theta} . .
\end{aligned}
$$

Lynde's results for three pairs of partially miscible liquids, together with the calculated values of $h, \Gamma_{0(1)}$, and

\begin{tabular}{|c|c|c|c|c|c|c|}
\hline $\mathrm{C}_{0}$. & $\mathrm{O}_{1}$. & $\stackrel{\text { in dyne/cm. }}{\text { ing }}$ & $\begin{array}{c}\frac{d \tau}{d p} \\
\text { in } \mu \mu .\end{array}$ & $\begin{array}{c}h \\
\text { in } \mu \mu .\end{array}$ & $\begin{array}{c}\Gamma_{0(1)} \\
\text { in } 10^{-\mathbf{s}} \mathrm{gm} . / \mathrm{cm} .\end{array}$ & $\begin{array}{c}\Gamma_{\mathbf{1}(0)} \\
\text { in } 10^{-8} \mathrm{gm} \cdot / \mathrm{cm}^{2}\end{array}$ \\
\hline Water. & Ether. & $9 \cdot 7$ & -.060 & -.068 & $+\cdot 6$ & $+\cdot 4$ \\
\hline Water. & Chloroform. & 27 & -.006 & -.006 & $+\cdot 06$ & +.09 \\
\hline Water. & $\begin{array}{l}\text { Carbon bi- } \\
\text { sulphide. }\end{array}$ & 42 & $+\cdot 037$ & $+\cdot 041$ & $-\cdot 4$ & $-\cdot 5$ \\
\hline
\end{tabular}
$\Gamma_{1(0)}$ are shown in the following table.

It will be seen that in two cases $d \tau / d p$ is negative. The distance between the "zero-planes" is, however, much too small to justify any assumption of surface compression.

Case III. Liquid Pbase in contact with Vapour Phase (both components volatile).

In this case we may in general neglect the densities in the vapour phase in comparison with those in the liquid phase. Phil. Mag. S. 6. Vol. 31. No. 184. April 1916. U 
Equation (10) then becomes

or

$$
\begin{aligned}
& \frac{\Gamma_{1}}{\rho_{1}}-\frac{\Gamma_{0}}{\rho_{0}}=\left(\frac{\rho_{0}^{\prime}}{\rho_{0}}-\frac{\rho_{1}^{\prime}}{\rho_{1}}\right)\left(\frac{d \tau}{d p}\right)_{\theta} \cdot . . \\
& \Gamma_{1(0)}=\left(\frac{\rho_{1} \rho_{0}^{\prime}}{\rho_{0}}-\rho_{1}^{\prime}\right)\left(\frac{d \tau}{d p}\right)_{\theta} . .
\end{aligned}
$$

If we suppose the vapour phase to consist of an ideal mixture of gases of molecular weights $m_{0}$ and $m_{1}$ respectively, the above equation reduces to

$$
\Gamma_{1(0)}=\frac{\rho_{1}}{\mathrm{R} \theta}\left(\frac{m_{0} p_{0}}{\rho_{0}}-\frac{m_{1} p_{1}}{\rho_{1}}\right)\left(\frac{d \tau}{d p}\right)_{\theta}, . . .
$$

where $\mathrm{R}$ is the "gas constant," and $p_{0}$ and $p_{l}$ the partial pressures of the vapours of $\mathrm{C}_{0}$ and $\mathrm{C}_{1}$ respectively. If we suppose the state of the system to be specified by the concentration $c$ of the solution of $\mathrm{C}_{1}$ in $\mathrm{C}_{0}$ we may write

$$
\Gamma_{1(0)}=\frac{\rho_{1}}{\operatorname{R} \theta}\left(\frac{m_{0} p_{0}}{\rho_{0}}-\frac{m_{1} p_{1}}{\rho_{1}}\right) \frac{\left(\frac{d \tau}{d c}\right)_{\theta}}{\left(\frac{d p}{d c}\right)_{\theta}} . . .
$$

The concentration $c$ may be defined in any of the three usual ways

$$
\begin{aligned}
& c=\rho_{1}, \\
& c=\frac{\rho_{1}}{\rho_{0}},
\end{aligned}
$$

and

$$
c=\frac{\rho_{1}}{\rho_{0}+\rho_{1}} .
$$

Let us now consider the case of an ideal dilute solution of $\mathrm{C}_{1}$ in $\mathrm{C}_{0}$. In such a solution the partial pressure of the solute is proportional to its concentration, and the relative lowering of the pressure of the solvent is equal to the "molar fraction" of the solute. We have therefore

$$
p_{1}=\mathrm{K} \rho_{1}, \quad \cdot \text {. . . . . }
$$

where $K$ is some constant * about the magnitude of which

- Or more precisely, some function of the temperature. 
the Theory of the Ideal Dilute Solution makes no statement; and

$$
\frac{\mathrm{P}_{0}-p_{0}}{\mathrm{P}_{0}}=\frac{m_{0} \rho_{1}}{m_{1} \rho_{0}}, \ldots \text {. . . . . . }
$$

where $P_{0}$ is the vapour-pressure of the pure solvent. Substituting the values of $p_{0}, p_{1}$, and $d p$ given by equations (25) and (26) in equation (23) we obtain the result

$$
\Gamma_{1(0)}=-\frac{m_{1} \rho_{1}}{\mathrm{R} \theta}\left(\frac{d \tau}{d \rho_{1}}\right)_{\theta}, \quad . \quad . \quad . \quad .
$$

which differs from the well-known result deduced by Milner * only in respect to the more precise definition of the "surface excess." We thus see that the equation applies to the case of a dilute solution of a volatile substance in contact with the vapour phase.

We will consider the question of liquid mixtures in Part II., when we deal with the case of a solution in contact with a third substance. Practically all the experimental data available for illustrating the theory relate to this latter case, which is a special case of a ternary system.

Case IV. Liquid Phase in contact with Vapour Phase (only one component volatile).

If $\mathrm{C}_{1}$ is involatile equation (22) becomes

$$
\Gamma_{1(0)}=\frac{\rho_{\mathrm{t}} \rho_{0}^{\prime}}{\rho_{0}}\left(\frac{d \tau}{d p}\right)_{\theta} \cdot \cdot \cdot \cdot \cdot \cdot
$$

If the solvent vapour behaves as an ideal gas of molecular weight $m_{0}$, we have

$$
\Gamma_{1(0)}=\frac{m_{\mathrm{o}} \rho_{1} p}{\mathrm{R} \theta}\left(\frac{d \tau}{d p}\right)_{\dot{\theta}} \cdot \cdot \cdot . \cdot .
$$

In the case of an ideal dilute solution equation (27) is obviously valid.

The University of Leeds.

Feb. 3rd, 1916.

* Loc. eit. 